\title{
Low dose oral methotrexate treatment of multiple sclerosis: a pilot study
}

\author{
Robert D Currier, Armin F Haerer, Edward F Meydrech
}

\begin{abstract}
An 18-month double-blind treatment of multiple sclerosis with low dose oral methotrexate showed it to be well tolerated and suggested effectiveness in exacerbating-remitting MS but not in the exacerbating progressive and chronic progressive stages.
\end{abstract}

$(\mathcal{F}$ Neurol Neurosurg Psychiatry 1993;56:1217-1218)

Multiple sclerosis (MS) has been treated with immunological altering methods and drugs for the past two decades. Some appear to be beneficial but problems with administration, cost, side effects, the need for laboratory studies, delayed effectiveness, poor patient acceptance and compliance and the possibility of remote cancer, have limited their use.

Methotrexate in low dose format has been used to treat psoriasis and rheumatoid arthritis for four decades. It may be the most effective and least harmful treatment for progressive rheumatoid arthritis. ${ }^{1}$ A trial of low dose methotrexate for MS seemed reasonable. Oral methotrexate was previously evaluated by Neumann and $Z_{\text {iegler }}^{2}$ who gave 15 patients $2.5 \mathrm{mg}$ of oral methotrexate daily alternating with 6-mercaptopurine in threemonth time periods over 10 to 24 months. When compared with a placebo group no obvious difference was noted.

\section{Method}

Approval for the trial was given by the University Human Investigation Committee.

University of Mississippi Medical Center, Jackson, MS, USA

Department of

Neurology

R D Currier

A F Haerer

Department of

Preventive Medicine

E F Meydrech

VA Medical Center, Jackson, MS, USA A F Haerer

Correspondence to: Dr Currier, Department of Neurology, University of Mississippi Medical Center, Jackson, MS 39216, USA.

Received 6 May 1992 and in final revised form 23 December 1992 Accepted 14 January 1993
One of us (AFH) acted as the 'blind' evaluator and the other (RDC) as the treating physician who might if necessary break the 'blind', a need that occurred twice. The patients were randomised as they entered the study.

The medication was given as one tablet ( $2.5 \mathrm{mg}$ methotrexate or placebo) every 12 hours for three consecutive doses, once a week $(7.5 \mathrm{mg} /$ week); the usual treatment regime for rheumatoid arthritis. Complete blood counts, complete blood chemical analyses and chest radiographs were carried out at the beginning and end of the study. Blood counts and liver function studies were done every two months. Neurological examinations were done at entry, every four months and at the end, and graded on the Kurtzke ${ }^{3}$ scale. Additional treatments such as corticosteroids were allowed and were given for exacerbations during the study.

Forty-five patients were enrolled for a period of a year. One took a single pill and dropped out. The remaining 44 were included in the analysis. The time in the study of the six who stopped the medication before the 12 month point was included. At the end of the study the 'blinded' physician categorised the patients into subtypes of MS and tabulated the number and timing of exacerbations and worsenings by one point or more on the Kurtzke scale. An exacerbation

Time of event, months

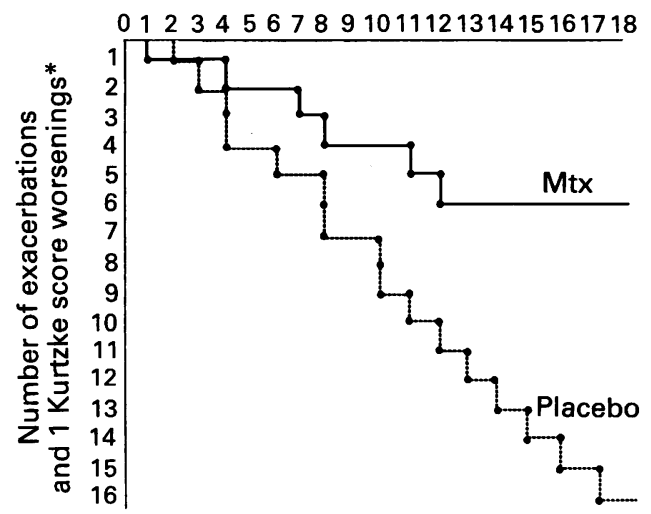

Figure Exacerbations and worsenings of multiple sclerosis of one Kurtzke grade or more in the exacerbating-remitting methotrexate group (nine patients) and the placebo group (11 patients). *If a patient had both an exacerbation and a Kurtzke worsening in the same month, it was counted as a single event. 
lasting at least two weeks followed by incomplete or complete recovery and so judged by an experienced neurologist.

Fisher's Exact Test was used to look for an association between the placebo and methotrexate treatments and the proportion of subjects having exacerbations. The twosample $t$ test was used to compare the mean difference in the number of exacerbations between the treatment and placebo groups and to compare the mean difference in the number of exacerbations plus Kurtzke worsenings greater than one between the methotrexate and placebo portions of the exacerbating-remitting group.

\section{Results}

When considered as a single group there was no difference in outcome of the 22 methotrexate and 22 placebo patients. Eight on methotrexate and nine on placebo had exacerbations. Of the 44, 20 had exacerbating-remitting MS and 24 chronic MS including 18 exacerbating progressive, three chronic progressive and three spinal patients. In the exacerbating-remitting group of 20 (11 placebo, nine methotrexate) there were 14 females and six males, median age 39.5 years. In the 12 months before the study there were 12 exacerbations in the 11 placebo patients and nine in the nine methotrexate patients. During treatment a total of three exacerbations occurred in two of the nine exacerbating-remitting methotrexate patients over a period of 150 treatment months, for a mean of one exacerbation per 50 months. Seven of the 11 placebo patients had 12 exacerbations during 169 total group treatment months, a mean of one per 14 months. The difference in proportions of patients having exacerbations is not significant $(p=0.09)$ while the difference in the mean number of exacerbations just reaches significance with $p=0 \cdot 05$. Three of the nine methotrexate patients and five of 11 placebo patients worsened by one or more points on the Kurtzke scale.

The figure shows the time of exacerbations and worsenings of one Kurtzke grade or more in the exacerbating-remitting methotrexate and placebo groups. The placebo group had 16 events which continued to occur to the end of the study (three placebo patients experienced an exacerbation and a Kurtzke grade worsening simultaneously which were considered as one event in each case) and the methotrexate group had six events, with no worsenings after 12 months. The difference in the mean number of exacerbations plus Kurtzke score worsenings of one or more is just significant with $\mathrm{p}=0.05$.

There were 24 patients (11 placebo, 13 methotrexate) in the chronic MS group, 19 female and five male, median age 46.8 years. The three early dropouts at four, five and eight months were all on placebo. Total treatment times were 156 months for placebo and 230 for methotrexate.

Two of 11 patients on placebo had a total of three exacerbations in 156 months of treatment, one per 52 months, and six of 13 methotrexate patients had a total of nine exacerbations in 230 months of treatment, or one every 25.5 months. Two of 13 methotrexate patients worsened by one or more points on the Kurtzke scale and none of the 11 placebo patients worsened by one point or more.

While none of the outcome variables nears statistical significance in the chronic MS group ( $p=0.15$ for the difference in proportions with exacerbations and $p=0.11$ for the difference in the number of exacerbations), it may be important to notice that the direction of the differences is opposite to the exacerbating-remitting group.

The methotrexate was tolerated reasonably well. Two patients complained of headaches, both were on placebo. Nausea was helped by taking the medication with meals. One methotrexate patient had moderate hair thinning. Liver enzymes became mildly to moderately abnormal on more than one occasion in eight methotrexate and one placebo patients but were persistently abnormal in only one methotrexate patient. In that patient the medication was stopped, the enzymes returned to normal and the medication was restarted with the enzymes remaining normal.

\section{Discussion}

The effect of methotrexate on the liver has been a concern over the years. It is proving to be less toxic than originally thought, and the rheumatologists, the chief users of oral low dose therapy, are gradually using fewer tests of hepatic function. In a recent five year study of low dose methotrexate in the treatment of rheumatoid arthritis, ${ }^{4} 44$ of 124 patients stopped the treatment because of side effects including nausea, stomatitis, hair loss, rash, pulmonary reactions, elevated liver enzymes, haematological abnormalities and hepatic fibrosis. Although $93 \%$ of the total experienced at least one drug reaction the majority did not require permanent drug discontinuation. There is no evidence that low dose methotrexate causes cancer.

In the present study those with exacerbating-remitting MS apparently benefited and those with chronic MS worsened. Why did not the methotrexate benefit both types? The question of dosage is debatable.

This is a small trial and must be considered a preliminary study of low dose oral methotrexate in the treatment of multiple sclerosis. Methotrexate therapy is now under evaluation by others with larger and longer studies which should help determine the truth of the matter.

Lederle Pharmaceuticals kindly provided the methotrexate and placebo for this study and helped defray the cost of the laboratory work.

1 Wilke WS, ed. Methotrexate therapy in rheumatic disease New York: Marcel Dekker, 1989.

2 Neumann JW, Ziegler DK. Therapeutic trial of immunosuppressive agents in multiple sclerosis. Neurology 1972 ; suppressive

3 Kurtzke JF. A new scale for evaluating disability in multiple sclerosis. Neurology 1955;5:580-3.

4 Scully DJ, Anderson CJ, Cannon CW. Long-term methotrexate therapy for rheumatoid arthritis. Seminar in Arthritis and Rheumatism 1991;20(5):317-31. 
There are also practical and concise chapters on operative positioning and perioperative management, and on cranial nerve function monitoring.

The scope of the book is therefore very wide, but-no criticism intended-those seeking information on vascular lesions or intra-axial tumours should look elsewhere. The text is invariably practical and authoritative, and the illustrations uniformly good. I could not fault the index.

This book is probably a must for departmental libraries, and will be welcomed by all neurosurgeons undertaking surgery in the posterior fossa, and perhaps particularly by those preparing to sit specialty Fellowship.

AJ STRONG

Cerebral Small Artery Disease. (Advances in Neurology, Vol 62). Edited by PM PULLICINO, LR CAPLAN and M HOMMEL (Pp 241, Illustrated; Price: \$123.50). 1993 New York, Raven Press. ISBN 0-78170051-5 (Order Code 2591)

In the Middle Ages a favoured topic for debate among theologians concerned how many angels could rest on the point of a needle; latter-day neurologists debate with equal vigour (but no more success) the doctrine of the lacune. These perplexing holes were first well described by French pathologists as small cavities within the substance of the brain and thought by most to be ischaemic in origin. Other pathological conditions resembling but distinct from lacunes were recognised and the term came to be used to describe small circumscribed deep-seated infarcts. Early attempts to correlate individual lacunes with clinical symptoms and signs were hampered by the fact that there was no way of locating with certainty the site of lesion during life and when the patient eventually came to autopsy the lacunes were usually multiple. Pierre Marie however recognised the clinical correlate of longstanding état lacunaire as a pseudobulbar palsy, spastic quadriparesis and dementia.

In the 1960s Fisher using a classical clinico-pathological approach defined a group of clinical syndromes (pure motor stroke, ataxic hemiparesis, dysarthriaclumsy hand syndrome) caused by small deep infarcts in specific locations. It soon became clear that the same syndrome could result from a lacune in a number of different sites, that a clinical lacunar syndrome did not always signify a small deep-seated lesion and that pathological lacunes could be found in the absence of any symptoms.

In recent years modern imaging has opened up the possibility of clinicopathological correlation during life. However radiolr gical changes may be deceptive-not all regions of signal change are infarcts, the lesions are often multiple and the size of the lesion on imaging (an important diagnostic criterion) may differ greatly from its pathological size.

We end up with three definitions of lacunes-pathologists mean a small deep infarct in certain sites, clinicians mean one of a number of neurological syndromes and radiologists mean a region of signal change of a specified size and location. The possibilities for argument and misunderstanding are endless.

As if this were not enough, the disagreement about the nature of a lacune has developed into a further argument about its cause. Modern views on this have again been much influenced by Fisher who regarded a lacune as a small deep infarct exclusively in the territory of a penetrating artery. In most cases the penetrating artery is occluded by lipohyalinosis, a consequence of chronic hypertension, but in a few occlusion results from atheroma or embolism. Furthermore the artery is not always completely blocked and it now appears that small deeply placed infarcts in distal field territories can be caused by haemodynamic events such as systemic hypotension in patients with occlusions of large extracranial arteries.

This well-produced book deals extensively with these controversies and leaves the reader (like FE Smith's judge) possibly none the the wiser but certainly better informed. There are excellent reviews of cerebrovascular anatomy, microvascular territories, arterial pathology, radiology and clinical syndromes and much speculation on pathogenesis, notably a well-balanced and informative chapter from Dr Pullicino, one of the editors. The illustrations are also unusually good and the chapters are fully referenced. All in all this is a book to be recommended to all those interested in cerebral vascular disease and one which shines some welcome light into dark places and small holes.

R ROSS RUSSELI

The Biology of the Autistic Syndromes/2nd Edition. By C GILLBERG and $M$ Coleman. (Pp 317, Illustrated; Price $\$ 37.50) .1992$ Cambridge University Press. ISBN 0-521-43228-6.

This is a remarkable book. No one who deals with autism will ever be the same after reading this book which is a fresh edition of the original title by Coleman and Gilberg published by Praeger New York in 1985. In scope and detail it is as rich as an oldfashioned Christmas cake, not to mention the hidden charms which may cause injury if not noticed. In so far as the book is aimed not only at psychiatrists and neurologists but also at psychologists special educators and even parents of children with autism, it is inevitable that which components are indigestible will vary from one readership group to another.

Originally there was Kanner's infantile autism but that has not been a helpful concept nor has the idea of "pervasive developmental disorder": autism is a specific neurological symptom complex not nearly as pervasive as in less politically correct times was called mental deficiency or mental retardation. Having teased that out the authors review the clinical, epidemiological, genetic, biochemical, endocrine, immunological, electrophysiological, brain imaging, neuropathological and neuropsychological literature with extensive references.

I have concerns that child psychiatrists who read this book may do the recommended neurological examination and the recommended investigations without the intermediary of a paediatric neurologist, a difficulty being that the authors are not neurologists themselves. My substitution for the second part of table 25.1 (neuropsychiatric assessment check list for autism) would be first to get a first quality paediatric neurologist to conduct the examination (for example one doesn't just look for Lisch nodules as confirmation of type I Neurofibromatosis - one arranges for a slitlamp by the ophthalmologist). You may investigate using the philosophy of testing outlined in Handbook of Neurological Investigations in Children. This table ends with a novel inverted definition of the milkmaid sign.

There are lucky charms in this book as for example in the section on epilepsy. There one finds for example a fine reference to "autisme convulsi?" to emphasise that not only do epilepsy and autism go together but that autism might be a symptom of the epileptic process, the manifestation of one type of minor epileptic status. This is an autism treatable by anti-epileptic medication. There is uncontrolled evidence that vigabatrin when it abolishes infantile spasms may reduce subsequent autism liability.

J B P STEPHENSON

SHORT NOTICES

Electrical and Magnetic Stimulation of the Brain and Spinal Cord (Advances in Neurology, Vol. 63). Edited by O DEVINSKY, A BERIC AND MICHAEL DOGALI. (Pp 323; Price: \$139.00) 1993. New York, Raven Press. ISBN 0-7817-0066-3.

Anatomic Localization for Needle Electromyography. By STEVE R REIRINGER. (Pp 154 Illustrated; Price: \$28.95) 1994 Philadelphia, Hanley \& Belfus Inc. ISBN 1-56053-068-5.

\section{CORRECTION}

Currier RD, Haerer AF, Meydrech, EF. Low dose oral methotrexate treatment of multiple sclerosis: a pilot study. $\mathcal{f}$ Neurol Neurosurg Psychiatry 1993;56:1217-18. The final sentence on p.1217 should read "An exacerbation was defined as a focal neurological worsening lasting at least two weeks followed by incomplete or complete recovery and so judged by an experienced neurologist." 